\title{
242.
}

\section{SECOND NOTE ON POINSOT'S FOUR NEW REGULAR POLYHEDRA.}

[From the Philosophical Magazine, vol. xvII. (1859), pp. 209-210.]

The Note on Poinsot's four new regular Polyhedra (February Number, p. 123), [241], was written without my being acquainted with Cauchy's first memoir, "Recherches sur les Polyèdres" (Jour. Polyt. vol. Ix. pp. 68-86, 1813), the former part of which (pp. 68-76) relates to Poinsot's polyhedra. Cauchy considers the polyhedra, not as projected on the sphere, but in eolido; and he shows, very elegantly, that all such polyhedra must be derived from the ordinary regular polyhedra by producing their sides or faces. The reciprocal method would be to produce the sides or join the vertices; and, adopting this reciprocal method, and projecting the figure on the sphere, we have the method employed by Poinsot, and explained and developed in my former Note. Cauchy does not at all consider Poinsot's generalized equation, $e S+H=A+2 E$, nor of course my further generalization, $e S+e^{\prime} H=A+2 D$; but the latter part of the memoir relates to a generalization, in a different direction, of Euler's original formula, $S+H=A+2$ : viz. Cauchy's theorem is- "If a polyhedron is partitioned into any number of polyhedra by taking at pleasure, in the interior of it, any number of new vertices, and if $P$ be the total number of polyhedra thus formed, $S$ the total number of vertices (including those of the original polyhedron), and $A$ the total number of edges, then $S+H=A+P+1$; that is, the sum of the number of vertices and the number of faces exceeds by unity the sum of the number of edges and of the number of polyhedra."

For $P=1$, we have Euler's equation $S+H=A+2$; and for $P=0$, we have a theorem relating to the partition of a polygon; viz. if the polygon is divided into $H$ polygons, and if $S$ be the number of vertices, and $A$ the number of sides, then $S+H=A+1$; from which it is easy to pass to Euler's equation, $S+H=A+2$, for 
polyhedra. I remark that, in the equation $S+H=A+1, H$ should, in analogy with Cauchy's notation for polyhedra, be replaced by $P$; so that we have for a single polygon,

$$
A=S \text {; }
$$

and for the partitions of a polygon,

$$
A=S+P-1:
$$

corresponding respectively to Euler's theorem for a single polyhedron, viz.

$$
S+H=A+2 \text {; }
$$

and to Cauchy's theorem for the partitions of a polyhedron, viz.

$$
S+H=A+2+(P-1) \text {. }
$$

Cauchy's second memoir (pp. 87-98) contains a very beautiful demonstration of the theorem implied in the ninth definition of the eleventh book of Euclid, viz. that two convex polyhedra are equal when they are bounded by the same number of faces equal each to each.

2, Stone Buildings, W.C., February 1, 1859. 